\title{
advances in genomic sequence analysis and pattern discovery
}




\section{SCIENCE, ENGINEERING, AND BIOLOGY INFORMATICS}

Series Editor: Jason T. L. Wang

(New Jersey Institute of Technology, USA)

Published:

Vol. 1: Advanced Analysis of Gene Expression Microarray Data (Aidong Zhang)

Vol. 2: $\quad$ Life Science Data Mining (Stephen T. C. Wong \& Chung-Sheng Li)

Vol. 3: Analysis of Biological Data: A Soft Computing Approach (Sanghamitra Bandyopadhyay, Ujjwal Maulik \& Jason T. L. Wang)

Vol. 4: Machine Learning Approaches to Bioinformatics (Zheng Rong Yang)

Vol. 5: Biodata Mining and Visualization: Novel Approaches (Ilkka Havukkala)

Vol. 6: Database Technology for Life Sciences and Medicine (Claudia Plant \& Christian Böhm)

Vol. 7: Advances in Genomic Sequence Analysis and Pattern Discovery (Laura Elnitski, Helen Piontkivska \& Lonnie R. Welch) 


\title{
advances in genomic sequence analysis and pattern discovery
}

\author{
editors \\ Laura Elnitski \\ National Human Genome Research Institute, \\ National Institutes of Health, USA \\ Helen Piontkivska \\ Kent State University, USA \\ Lonnie R Welch \\ Ohio University, USA
}


Published by

World Scientific Publishing Co. Pte. Ltd.

5 Toh Tuck Link, Singapore 596224

USA office: 27 Warren Street, Suite 401-402, Hackensack, NJ 07601

UK office: 57 Shelton Street, Covent Garden, London WC2H 9HE

\title{
British Library Cataloguing-in-Publication Data
}

A catalogue record for this book is available from the British Library.

\author{
ADVANCES IN GENOMIC SEQUENCE ANALYSIS AND PATTERN DISCOVERY \\ Science, Engineering, and Biology Informatics - Vol. 7 \\ Copyright () 2011 by World Scientific Publishing Co. Pte. Ltd. \\ All rights reserved. This book, or parts thereof, may not be reproduced in any form or by any means, \\ electronic or mechanical, including photocopying, recording or any information storage and retrieval \\ system now known or to be invented, without written permission from the Publisher.
}

For photocopying of material in this volume, please pay a copying fee through the Copyright Clearance Center, Inc., 222 Rosewood Drive, Danvers, MA 01923, USA. In this case permission to photocopy is not required from the publisher.

ISBN-13 978-981-4327-72-5

ISBN-10 981-4327-72-7

Typeset by Stallion Press

Email: enquiries@ stallionpress.com

Printed in Singapore. 


\section{Contents}

Preface vii

About the Editors $\quad$ ix

Part I: Pattern Discovery Methods 1

Chapter 1: Large-Scale Gene Regulatory Motif Discovery

with NestedMICA

Matias Piipari, Thomas A. Down

and Tim J. P. Hubbard

Chapter 2: R'MES: A Tool to Find Motifs with a Significantly

Unexpected Frequency in Biological Sequences

Sophie Schbath and Mark Hoebeke

Chapter 3: An Intricate Mosaic of Genomic Patterns

at Mid-range Scale

Alexei Fedorov and Larisa Fedorova

Chapter 4: Motif Finding from Chips to ChIPs

Giulio Pavesi

Chapter 5: A New Approach to the Discovery of RNA

Structural Elements in the Human Genome

Lei Hua, Miguel Cervantes-Cervantes

and Jason T. L. Wang

Part II: Performance and Paradigms

Chapter 6: Benchmarking of Methods for Motif

Discovery in DNA

Kjetil Klepper, Geir Kjetil Sandve, Morten Beck Rye, Kjersti Hysing Bolstad and Finn Drabløs 
Chapter 7: Encyclopedias of DNA Elements for Plant Genomes Jens Lichtenberg, Alper Yilmaz, Kyle Kurz, Xiaoyu Liang, Chase Nelson, Thomas Bitterman, Eric Stockinger, Erich Grotewold and Lonnie R. Welch

Chapter 8: Manycore High-Performance Computing in Bioinformatics Jean-Stéphane Varré, Bertil Schmidt, Stéphane Janot and Mathieu Giraud

Chapter 9: Natural Selection and the Genome Austin L. Hughes

Index 


\section{Preface}

Those who are involved with mapping the genomic landscapes are participating in one of the most exciting frontiers of science. We have the opportunity to reverse engineer the blueprints and the control systems of living organisms. Computational tools are key enablers in the deciphering process. Thus, this book provides an in-depth presentation of some of the important computational biology approaches to genomic sequence analysis. The first part of the book discusses methods for discovering patterns in DNA and RNA. This is followed by the second part that reflects on methods in various ways, including performance, usage and paradigms.

Part I, Pattern Discovery Methods, provides a collection of computational methods and tools. Chapter 1, "Large-Scale Gene Regulatory Motif Discovery with NestedMICA," presents an algorithmic approach, describes usage of the tool based on the algorithm, and illustrates its usage via a detailed case study. In Chapter 2, “R'MES: A Tool to Find Motifs with a Significantly Unexpected Frequency in Biological Sequences," the authors describe a software tool that contains rigorous statistical models of DNA words. "An Intricate Mosaic of Genomic Patterns at Mid-range Scale," Chapter 3 of the book, focuses on intricate mosaics found in genomes; a number of specific patterns are identified. The fourth chapter, "MotifFinding from Chips to ChIPs," provides a comprehensive survey of methods for the de novo discovery of putative over-represented transcription factor binding sites in nucleotide sequences. Part I concludes with a chapter that considers the discovery of RNA structural motifs: "A New Approach to the Discovery of RNA Structural Elements in the Human Genome."

The second part, Performance and Paradigms, consists of chapters that contemplate the effectiveness of relevant computational biology techniques. Chapter 6, "Benchmarking of Methods for Motif Discovery in $D N A$," presents a variety of metrics for assessing the performance of the class of methods described in Part I. In "Encyclopedias of DNA Elements for Plant Genomes," the application of methods is illustrated with case studies. The topic of scalable algorithmic approaches is considered in 
Chapter 8, "Manycore High-Performance Computing in Bioinformatics." Chapter 9, "Natural Selection and the Genome," discusses evolution of genomic sequences and the role that natural selection plays in directing genome evolution. It also provides a conceptual framework for better understanding of the evolutionary implications and insights that are generated through genomic sequence analyses, and emphasizes the critical role of purifying selection. 


\section{About the Editors}

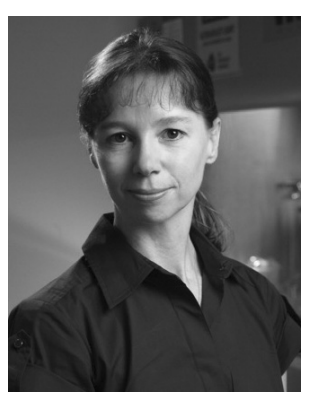

Dr. Laura Elnitski is a molecular and computational biologist who studies noncoding functional elements in vertebrate genomes. She has served as an analyst for the Mouse, Rat, Chicken and Bovine Genome Consortia.

Dr. Elnitski is extensively involved in NHGRI's ENCODE (Encyclopedia of DNA Elements) project, which aims to produce a comprehensive catalog of functional elements in the human genome. Dr. Elnitski's research uses integrative analyses to elucidate both the presence and activity of functional elements in the human genome that have been historically difficult to characterize. For example, computationally, her work predicts mutations in coding sequences that affect proper splicing. Targets of these mutations include exonic splicing enhancers and silencers. In experimental analyses, she is mapping elements that silence gene expression using an assay system designed in her lab.

Driving towards a molecular understanding of ovarian cancer, Dr. Elnitski combines in silico and wet-bench techniques. She has annotated bidirectional promoters in the human genome, including those regulating noncoding genes, using data collected in RNA-seq assays. These results are being used to find gene silencing events caused by aberrant methylation in tumor samples. She is also addressing functional consequences of mutations in those tumors.

Dr. Elnitski is the recipient of a Ruth L. Kirschstein Postdoctoral Fellowship through the NIH (2000-2003), Outstanding Research Achievement Award (International Symposium on Bioinformatics Research and Applications - 2007), a featured scientist in the Women in Bioinformatics Research documentary (2007) and a Genome Technology Young Investigator Award (2009). She serves as an ad hoc reviewer for the NIH GCAT Scientific Grant Review Panel and is an associate editor of BMC Genomics and formerly Genome Research. 
She is currently the Head of Genomic Functional Analysis Section of the Genome Technology Branch at the National Human Genome Research Institute, NIH, USA.

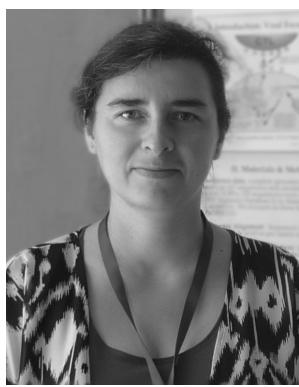

Dr. Helen Piontkivska is currently an Assistant Professor in the Department of Biological Sciences at Kent State University. She received her Ph.D. in Genetics from the Pennsylvania State University, and has over 10 years of experience in bioinformatics and evolutionary genomics and published over 35 peerreviewed publications. Dr. Piontkivska's research is focused on understanding the mechanisms of genome evolution using state-of-the-art bioinformatics approaches, in particular, delineating evolutionary mechanisms responsible for genomic changes in infectious agents, such as viruses, and disease-related genes, including cancer and immune and inflammatory genes using bioinformatics, machine learning and molecular evolutionary and phylogenetic approaches. Her work is funded by the National Institutes of Health. She currently serves on the editorial board of Molecular Biology and Evolution and is a member of the steering committee of The Ohio Bioinformatics Consortium.

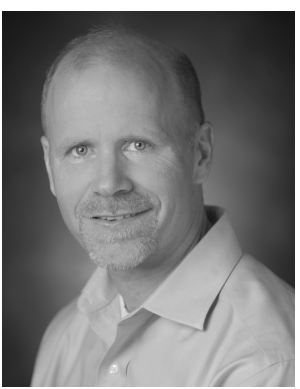

Professor Lonnie R. Welch received a Ph.D. in Computer and Information Science from the Ohio State University. Currently, he is the Stuckey Professor of Electrical Engineering and Computer Science at Ohio University, and he is a member of the Graduate Faculties of the Biomedical Engineering Program and of the Molecular and Cellular Biology Program.

Dr. Welch performs research in the areas of bioinformatics, computational regulatory genomics, machine learning and high performance computing. His research has been sponsored by the National Human Genome Research Institute, the Ohio Plant Biotechnology Consortium, NASA, the National Science Foundation, the Defense Advanced Research Projects Agency, and the Ohio Board of Regents. Dr. Welch has more than 20 years of research experience in the area of high performance computing. In his graduate work at Ohio State 
University, he developed high performance 3-D graphics rendering algorithms, and he invented a parallel virtual machine for object-oriented software. For 15 years, his research focused on middleware and optimization algorithms for high performance computing; this work produced three successive generations of adaptive resource management middleware for high performance real-time systems, and resulted in two patents and more than 150 publications. Currently, Professor Welch directs the Bioinformatics Laboratory at Ohio University, where he performs research in the area of computational regulatory genomics.

Dr. Welch is the founder and Chair of the Ohio Collaborative Conference on Bioinformatics and the Great Lakes Bioinformatics Conference (an official conference of the International Society on Computational Biology). As Founding Chair of the Ohio Bioinformatics Consortium, an Affiliated Regional Group of the International Society on Computational Biology, Dr. Welch has been an active member of the Regional Affiliates Committee of the ISCB. He is the Principal Investigator of the $\$ 9 \mathrm{M}$ Bioinformatics Program which is funded by the Ohio Board of Regents and 11 academic institutions from Ohio. Prof. Welch is founder and CoChair $(2010,2011)$ of the ISMB Special Interest Group on Bioinformatics for Regulatory Genomics (BioRegSIG). He has served on the organizing committees of the 2009 Bioinformatics Open Source Conference, the 2008 ISMB Special Interest Group on Genome-scale Pattern Analysis in the Post-ENCODE Era, the International Symposium on Bioinformatics Research and Applications, and the IEEE International Symposium on Bioinformatics and Bioengineering. 